\title{
Human CD83-targeted chimeric antigen receptor T cells prevent and treat graft-versus-host disease
}

\author{
Bishwas Shrestha, ${ }^{1}$ Kelly Walton, ${ }^{2}$ Jordan Reff, ${ }^{1}$ Elizabeth M. Sagatys, ${ }^{3,4}$ Nhan Tu, ${ }^{4}$ Justin Boucher, ${ }^{4}$ Gongbo Li, ${ }^{4}$ Tayyebb Ghafoor, ${ }^{4}$ \\ Martin Felices, ${ }^{2}$ Jeffrey S. Miller, ${ }^{2}$ Joseph Pidala, ${ }^{4,5}$ Bruce R. Blazar, ${ }^{6}$ Claudio Anasetti, ${ }^{4,5}$ Brian C. Betts, ${ }^{2}$ and Marco L. Davila ${ }^{1,4,5}$ \\ 'Department of Immunology, Moffitt Cancer Center, Tampa, Florida, USA. Division of Hematology, Oncology, and Transplantation, Department of Medicine, Masonic Cancer Center, University of Minnesota, \\ Minneapolis, Minnesota, USA. ${ }^{3}$ Department of Hematopathology and Laboratory Medicine, Moffitt Cancer Center, Tampa, Florida, USA. ${ }^{4}$ Department of Oncologic Sciences, University of South Florida, \\ Tampa, Florida, USA. ${ }^{5}$ Department of Blood and Marrow Transplantation and Cellular Immunotherapy, Moffitt Cancer Center, Tampa, Florida, USA. ${ }^{6}$ Division of Blood and Marrow Transplantation, \\ Department of Pediatrics, Masonic Cancer Center, University of Minnesota, Minneapolis, Minnesota, USA.
}

\begin{abstract}
Graft-versus-host disease (CVHD) remains an important cause of morbidity and mortality after allogeneic hematopoietic cell transplantation (allo-HCT). For decades, GVHD prophylaxis has included calcineurin inhibitors, despite their incomplete efficacy and impairment of graft-versus-leukemia (GVL). Distinct from pharmacologic immune suppression, we have developed what we believe is a novel, human CD83-targeted chimeric antigen receptor (CAR) T cell for CVHD prevention. CD83 is expressed on allo-activated conventional CD4 ${ }^{+} \mathrm{T}$ cells (Tconvs) and proinflammatory dendritic cells (DCs), which are both implicated in CVHD pathogenesis. Human CD83 CAR T cells eradicate pathogenic CD83 ${ }^{+}$target cells, substantially increase the ratio of regulatory T cells (Tregs) to allo-activated Tconvs, and provide durable prevention of xenogeneic GVHD. CD83 CAR T cells are also capable of treating xenogeneic GVHD. We show that human acute myeloid leukemia (AML) expresses CD83 and that myeloid leukemia cell lines are readily killed by CD83 CAR T cells. Human CD83 CAR T cells are a promising cell-based approach to preventing 2 critical complications of allo-HCT - GVHD and relapse. Thus, the use of human CD83 CAR T cells for CVHD prevention and treatment, as well as for targeting $C D 83^{+} \mathrm{AML}$, warrants clinical investigation.
\end{abstract}

\section{Introduction}

Allogeneic hematopoietic cell transplantation (allo-HCT) is a procedure performed with curative intent for high-risk hematologic malignancies and bone marrow failure syndromes. Annually, thousands of patients receive allo-HCT worldwide, and 34\%-89\% will develop acute graft-versus-host disease (GVHD) despite pharmacologic immune suppression $(1,2)$. The current practice is to use broadly suppressive calcineurin inhibitors combined with methotrexate, sirolimus, or mycophenolate mofetil to prevent GVHD. Despite known off-target impairment of beneficial graft-versus-leukemia (GVL) and limited tolerance induction (3), calcineurin inhibitors have been included in GVHD prophylaxis and treatment for more than 3 decades (4-6). While advances in donor and graft selection $(7,8)$, recipient comorbidity assessment $(9,10)$, and conditioning regimens have improved allo-HCT outcomes $(11,12)$, the use of calcineurin inhibitors remains prevalent in GVHD prevention (1). In particular, calcineurin inhibitors are still incorporated in the popular use of posttransplant cyclophosphamide-based regimens as GVHD prophylaxis (13).

Authorship note: MLD and BCB are co-senior authors who contributed equally to this manuscript.

Conflict of interest: MLD and BCB hold a provisional patent (WO2019165156) related to the use of the CD83 CAR T cell in GVHD prevention and treatment, "CD83-binding chimeric antigen receptors."

Copyright: ( 2020 , American Society for Clinical Investigation.

Submitted: December 16, 2019; Accepted: May 15, 2020; Published: July 27, 2020.

Reference information: J Clin Invest. 2020;130(9):4652-4662.

https://doi.org/10.1172/JCl135754.
Beyond calcineurin inhibitors, cell-based immune suppression is increasingly being studied in GVHD prevention. We and others have shown that Tregs offer potent and potentially antigenspecific inhibition of alloreactive T cells (14-16). Past clinical trials incorporating Tregs in GVHD prophylaxis have proven that cell-mediated immune suppression delivers safe and effective control over donor T cells without impairing GVL (17-19). Preclinical and clinical evidence also supports the translational potential of novel cell products, including natural killer (NK) cells, invariant NKT cells, myeloid-derived suppressor cells, and type 2 innate lymphoid cells to reduce GVHD and preserve GVL (20-25). Currently, these cell products remain largely investigational, though Tregs and NK cells have been widely studied in the clinical setting.

Recently, CAR T cells have demonstrated remarkable activity for treating relapsed/refractory acute lymphoblastic leukemia and diffuse large B cell lymphoma (26-29). Thus, FDA indications were given to CD19 CAR T cells for these hematologic malignancies. While CAR T cells are cytolytic and not inherently immune suppressive, they offer the potential to target cell mediators of GVHD. Moreover, in animal models we have demonstrated that CAR T cells carry a reduced capacity to elicit GVHD when administered after allo-HCT as a donor-derived product (30), providing a mechanism for the clinical observation that allogeneic CAR T cells do not mediate GVHD $(28,29)$.

CD83 represents a clinically relevant target to eliminate inflammatory DCs as well as alloreactive donor T cells. CD83 is a protein member of the immunoglobulin superfamily and is expressed on the surface of activated human DCs (31). Mature 
A

\begin{tabular}{|l|l|l|l|l|l|l|} 
VH & Linker & VL & CD8h/tm & 41BB & CD3 & GFP \\
\hline
\end{tabular}

B

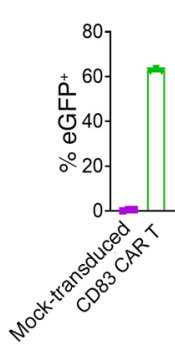

C

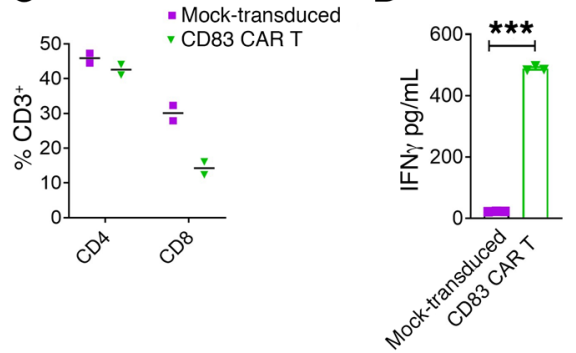

E
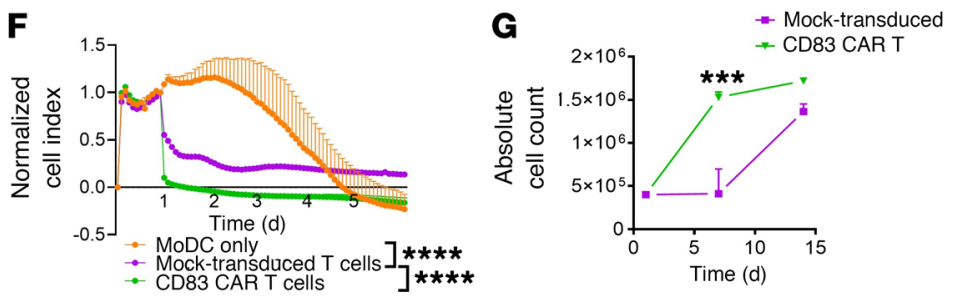

Figure 1. Human CD83-targeted CAR T construct and functional characteristics. (A) An anti-CD83 single-chain variable fragment is followed by a CD8 hinge and transmembrane domain, as well as a 41BB costimulatory domain and $C D 3 \zeta$ activation domain. The CAR is tagged with a fluorescence reporter at the $3^{\prime}$ end. The CAR reporter gene is cloned into an SFG retroviral vector. (B) Graph shows CAR gene transfer among T cells (mean \pm SEM) by expression of the intracellular EGFP reporter whereas mock-transduced cells are EGFP negative and CD83 CAR T cells are EGFP positive. (C) Graph demonstrates the relative amount of $\mathrm{CD} 4^{+}$or $\mathrm{CD} 8{ }^{+}$subsets among the mock-transduced or CD83 CAR T cells at day +7 after production ( $n=2-3$ independent donor experiments). ( $\mathbf{D}$ and $\mathbf{E}$ ) The amount of IFN- $\gamma$ and IL-2 released by mock-transduced or CD83 CAR T cells after stimulation with CD83 ${ }^{+}$DCs. (F) CD83 CAR T cells or mock-transduced T cells were cocultured with CD83 ${ }^{+}$ DCs and cytotoxicity was measured on a real-time cell analysis system. The data are presented (mean \pm SEM) as the average normalized cell index over time for duplicate wells. Normalized cell index is calculated as cell index at a given time point divided by cell index at the normalized time point, which is day 1 after addition of $\mathrm{T}$ cells. One representative experiment of 2 is shown. (C) CD83 CAR T cells or mock-transduced T cells were stimulated by CD83+ DCs and the absolute number of T cells (mean \pm SEM) was calculated weekly over a 14-day period. One representative experiment of 2 shown. ANOVA (D-G). ${ }^{* *} P=0.0001-0.001 ;{ }^{* * *} P<0.0001$

DCs can potentiate acute GVHD via allo-antigen presentation and proinflammatory cytokines (32). CD83 is also expressed on human $\mathrm{T}$ cells following stimulation by allo-antigen and is present on circulating T cells in patients with GVHD (31). Targeting CD83 with monoclonal antibody reduces xenogeneic GVHD in mice without impairing GVL or T cell responses against pathogenic viruses (33). However, the immune suppressive effect by the antibody is temporary and dependent on NK cell-mediated, antibody-dependent cellular cytotoxicity (ADCC) $(33,34)$.

Herein we describe the production and preclinical efficacy of human CD83-targeted CAR T cells for GVHD prevention and treatment. Unlike a monoclonal antibody, CD83 CAR T cells do not require ADCC to kill their targets. Moreover, we demonstrate that CD83 CAR T cells provide lasting GVHD prophylaxis in a human $T$ cell-mediated xenogeneic GVHD model after a single infusion of cells. Xenogeneic GVHD target organs exhibit essentially normal tissue within mice treated with CD83 CAR T cells. This is due in part to the differential expression of CD83 on activated conven- tional $\mathrm{CD} 4^{+} \mathrm{T}$ cells (Tconvs) versus Tregs. Thus, CD83 CAR T cells eliminate pathogenic Th1 cells and markedly increase the ratio of Tregs to Tconvs in vivo. CD83 CAR T cells also deplete proinflammatory $\mathrm{CD} 83^{+} \mathrm{DCs}$. Moreover, we demonstrate that human AML expresses CD83 and that myeloid leukemia is readily killed by CD83 CAR T cells. CD83 CAR T cells represent what we believe is an entirely novel, cell-based approach to GVHD therapy, and delivers durable, selective immune suppression while providing antitumor activity against $\mathrm{CD}^{+} 3^{+}$myeloid malignancies.

\section{Results}

Characterization of the human CD83 CAR T cell. To produce our human CD83-targeted CAR T cell, an anti-CD83 single chain variable fragment (scFv) was paired to a CD8 hinge and transmembrane domain, followed by the intracellular 41BB costimulatory domain and $\mathrm{CD} 3 \zeta$ activation domain (Figure $1 \mathrm{~A})$. To facilitate tracking of the CAR T cells, the construct contains an intracellular EGFP tag, which can be used to identify the CAR T cells among normal non-CAR T cells (Figure 1A). CD83-targeted CAR T cells were retrovirally transduced and generated as published (Figure $1 \mathrm{~A}$ and refs. 35,36 ).

The CD83 CAR construct exhibited a high degree of transduction efficiency, with more than $60 \%$ of $\mathrm{T}$ cells expressing the EGFP-tagged CAR construct (Figure 1B). While CD4 expression was similar among both groups, a reduction in CD8 expression was observed among CD83 CAR T cells compared with mock-transduced $\mathrm{T}$ cells (Figure 1C). However, the CD83 CAR T cells demonstrated robust IFN- $\gamma$ and IL-2 production when cultured with $\mathrm{CD} 83^{+}$target cells such as cytokine-matured human monocytederived DCs (moDCs) (Figure 1, D and E). Additionally, CD83 CAR T cells demonstrated potent killing of and proliferation against $\mathrm{CD} 83^{+}$moDCs compared with mock-transduced T cells (Figure 1, F and G). The target moDCs in these experiments were allogeneic to the $\mathrm{T}$ cells. Therefore, the lysis and proliferation by mock-transduced $\mathrm{T}$ cells represent baseline alloreactivity (Figure 1, F and G).

CD83 is differentially expressed on activated human Tconvs compared with Tregs. CD83 is an established marker of human DC maturation and is also expressed on activated human B cells (37, 38). Using a CD83 reporter mouse system, it was previously shown that activated murine $\mathrm{T}$ cells also express CD83 (39). It is known that CD83 is expressed on human T cells after stimulation and is detectable on circulating $\mathrm{T}$ cells from patients with acute GVHD (31). However, the precise expression of $\mathrm{CD} 83$ on $\mathrm{CD} 4^{+}$Tregs versus $\mathrm{CD} 4^{+}$Tconvs or $\mathrm{CD} 8^{+} \mathrm{T}$ cells is unclear. We confirmed that human $\mathrm{T}$ cell expression of CD83 occurs with stimulation, including allogeneic DCs or CD3/CD28 beads (Figure 2, $\mathrm{A}$ and B). Importantly, we demonstrate that $\mathrm{CD} 83$ is differentially expressed on human $\mathrm{CD}^{+}$Tconvs $\left(\mathrm{CD} 127^{+}, \mathrm{CD} 25^{+}\right)$compared with immune suppressive $\mathrm{CD}^{+}$Tregs $\left(\mathrm{CD} 127, \mathrm{CD}^{-} 5^{+}\right.$, $\left.\mathrm{Foxp}^{+}\right)$or cytolytic $\mathrm{CD} 8^{+} \mathrm{T}$ cells in response to DC alloactivation (Figure $2 \mathrm{~A}$ ). $\mathrm{CD}^{+}$ 
A

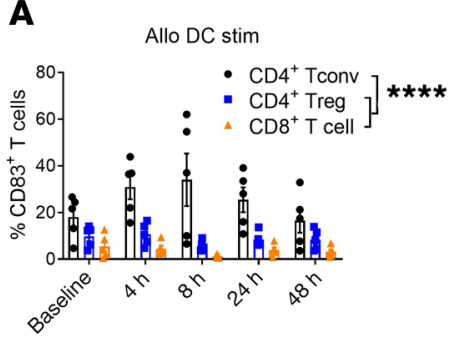

B

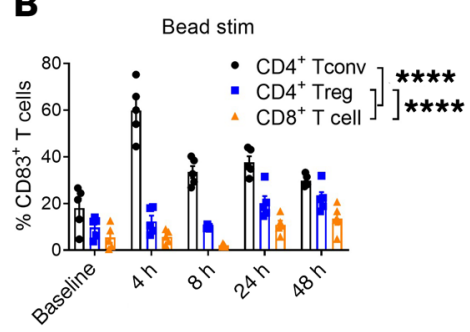

C

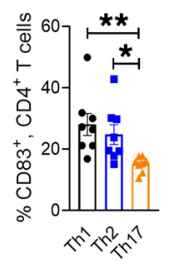

D

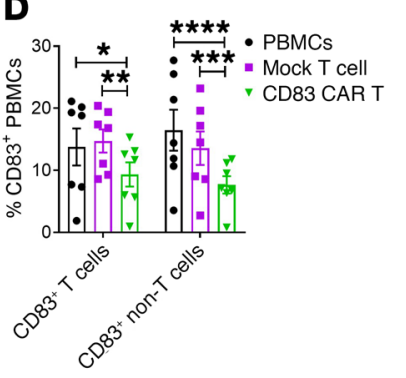

\section{E} PBMCs + AlloDCs + CD83 CAR

\section{8}
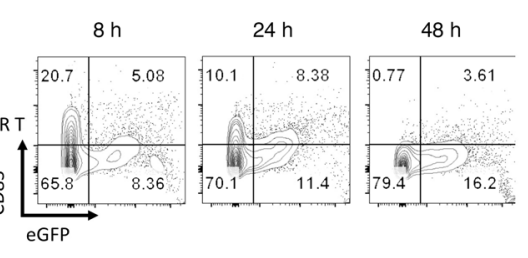

$\mathbf{F}$

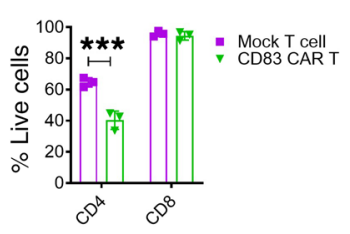

Figure 2. CD83 is differentially expressed on human activated conventional CD4 ${ }^{+} \mathrm{T}$ cells compared with regulatory $\mathrm{T}$ cells. Human $\mathrm{T}$ cells were stimulated by allogeneic moDCs (DC/T cell ratio, 1:30) or CD3/CD28 beads (bead/T cell ratio, 1:30). CD83 expression on activated Tconvs (CD4 ${ }^{+}, \mathrm{CD}_{127^{+}}$, CD25 $5^{+}$) or Tregs $\left(\mathrm{CD}^{+}, \mathrm{CD} 127^{-}, \mathrm{CD25} 5^{+}, \mathrm{Foxp3}^{+}\right)$was measured at baseline, 4 hours, 8 hours, 24 hours, and 48 hours after stimulation. Graphs show the amount of CD83 ${ }^{+} \mathrm{Tcon}^{-}$ vs or Tregs (mean \pm SEM) after (A) allogeneic DC or (B) CD3/CD28 bead stimulation ( $n=5$ independent experiments). (C) Graph shows the frequency (mean

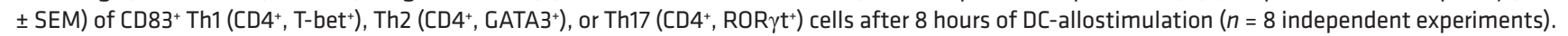
Human CD83 CAR or mock T cells were cultured with DC-allostimulated PBMCs at a ratio of 1:10 over 48 hours. (D) Graph shows the frequency of CD83+, CD3+, and CD3 target cells when cultured with CD83 CAR or mock-transduced T cells ( $n=7$ independent experiments). (E) Contour plots show the expression of CD83 among EGFP+ ${ }^{+}$CAR T cells over time. One representative experiment of 2 is shown. (F) Human CD4 ${ }^{+}$or CD8 ${ }^{+}$T cells were activated with CD3/CD28 beads for 8 hours, then removed from the beads and cocultured with autologous CD83 CAR T cells or mock-transduced T cells for 24 hours (CAR T-to-T cell ratio, 5:1). Graph shows the triplicate mean \pm SEM of live CD4 $4^{+}$or $C D 8^{+} T$ cells at the end of culture. One representative experiment of 2 is shown. ANOVA (A-D and F). ${ }^{*} P<0.05,{ }^{* *} P=0.001-0.01 ;{ }^{* *} P=0.0001-0.001 ;{ }^{* * *} P<0.0001$.

Tconv expression of CD83 peaks at 4-8 hours of DC allostimulation and declines to baseline levels by 48 hours in vitro, with minimal amounts observed on Tregs or $\mathrm{CD}^{+} \mathrm{T}$ cells (Figure 2A). The expression of CD83 is more abundant with supraphysiologic CD3/CD28 bead stimulation, which also causes a late increase in CD83 expression on Tregs and $\mathrm{CD}^{+} \mathrm{T}$ cells by 48 hours of acti-

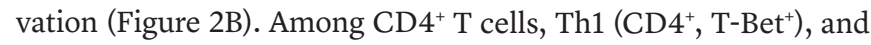
Th2 $\left(\mathrm{CD}^{+}, \mathrm{GATA}^{+}\right)$cells exhibit significantly increased CD83 expression compared with Th17s $\left(\mathrm{CD}^{+}, \mathrm{ROR} \gamma \mathrm{t}^{+}\right)$following DC allostimulation (Figure 2C). Given that CD83 expression is shared among proinflammatory mature DCs as well as alloreactive Tconvs, we investigated whether the CD83 CAR T cell could deplete either of these target cells in culture. Human CD83 CAR or mock $\mathrm{T}$ cells were cultured with autologous peripheral blood mononuclear cells (PBMCs) stimulated by allogeneic moDCs, and the amount of $\mathrm{CD} 83^{+}$target cells was evaluated at 8 hours of culture. CD83 CAR T cells significantly reduced the amount of $\mathrm{CD}^{2} 3^{+} \mathrm{T}$ cell and non-T cell targets in vitro (Figure 2D). Next, we evaluated the expression of CD83 on the $\mathrm{EGFP}^{+} \mathrm{CAR}$ T cells over 48 hours. CD83 expression on the CAR T cells was scant, and an increase in the proportion of $\mathrm{EGFP}^{+} \mathrm{CAR} \mathrm{T}$ cells was still observed by 48 hours of culture (Figure 2E), providing evidence that the CD83 CAR T cells do not overtly succumb to CD83-mediated fratricide. Consistent with the expression of CD83 on human T cells after 8 hours of polyclonal activation, the CD83 CAR T cells significantly killed CD3/CD28-bead stimulated $\mathrm{CD}^{+} \mathrm{T}$ cells but not $\mathrm{CD}^{+} \mathrm{T}$ cells in vitro (Figure 2F). Moreover, CD83- T cells were still present in all experimental groups (Figure $2 \mathrm{~F}$ ), supporting that $\mathrm{CD}^{-} 3^{-} \mathrm{T}$ cells are not indiscriminately destroyed.

Human CD83 CAR T cells reduce alloreactivity. To test whether human CD83 CAR T cells reduce alloreactivity in vitro, we investigated their suppressive function in allogeneic mixed leukocyte reactions (alloMLRs). CD83 and mock-transduced CAR T cells were generated from healthy donor human T cells. CD19 CAR T cells target B cells, an irrelevant cell type in the alloMLR, and were used as an additional control. CD19 and CD83 CAR T cells were similar in that they both received costimulation via 41BB. CAR or mock-transduced $\mathrm{T}$ cells were added to 5-day alloMLRs consisting of $\mathrm{T}$ cells $\left(1 \times 10^{5}\right)$ and allogeneic, cytokine-matured (40) $\mathrm{CD} 3^{+}$moDCs $\left(3.33 \times 10^{3}\right)$. The CAR $\mathrm{T}$ cell/moDC ratio ranged from 3:1 to 1:10. CD83 CAR T cells potently reduced proliferative alloreactive T cells (Figure 3). Conversely, mock-transduced and CD19-targeted CAR T cells had no suppressive effect against alloreactive $\mathrm{T}$ cells (Figure 3 ).

Human CD83-targeted CAR $T$ cells prevented xenogeneic GVHD. A xenogeneic GVHD model was used to evaluate the efficacy of human CD83 CAR T cells in vivo. We used our established NOD scid gamma (NSG) mouse model (41), in which recipients were inoculated with $25 \times 10^{6}$ human PBMCs plus either $1 \times 10^{6}$ to $10 \times 10^{6}$ autologous CD83 or mock-transduced CAR T cells all on day 0 . Transplanted mice were monitored daily for clinical signs of xenogeneic GVHD up to day +100 . NSG mice infused 


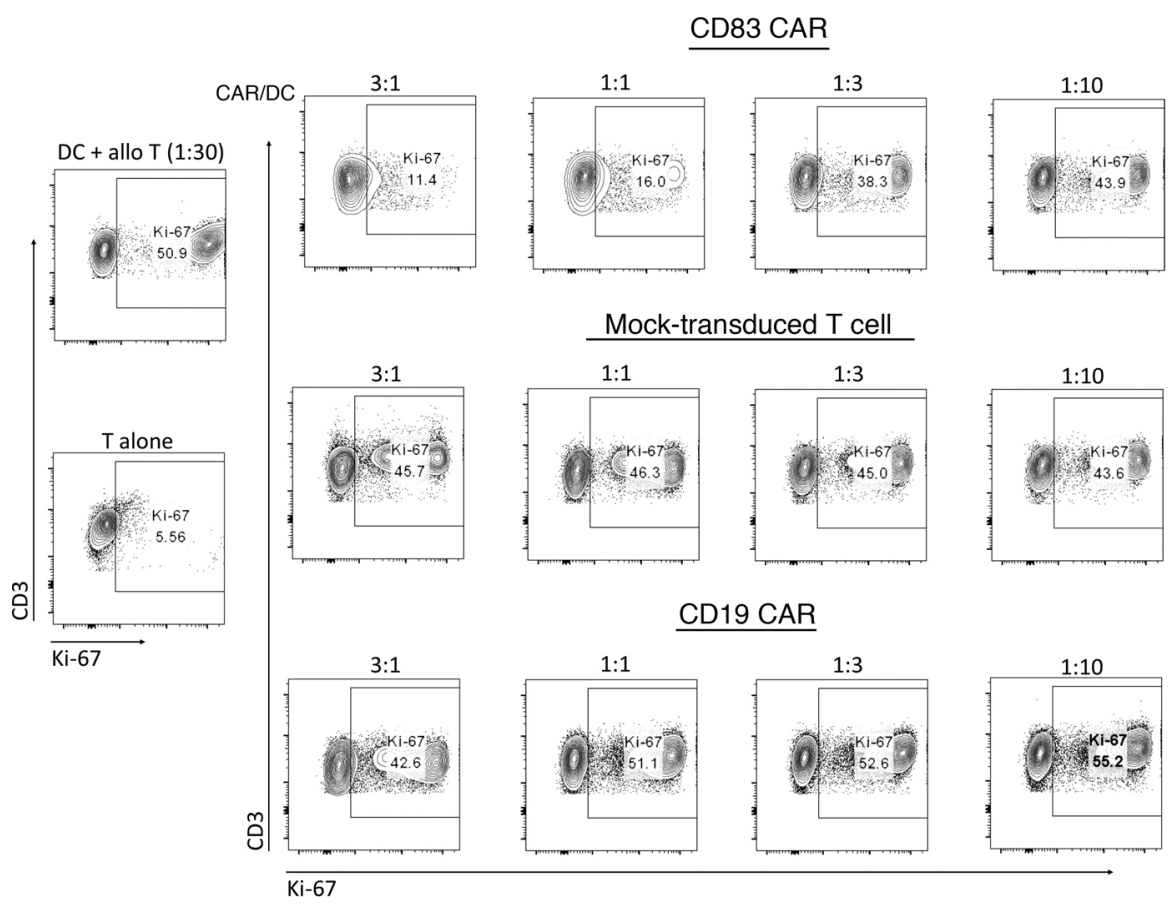

Figure 3. Human CD83 CAR T cells reduce alloreactivity. Human T cells were cultured with allogeneic cytokine-matured moDCs at a $D C / T$ cell ratio of 1:30 (i.e., 100,000 T cells and 3333 moDCs). CD83 CAR T cells were added at specific ratios to the moDCs (3:1 to 1:10, where the lowest amount of CAR T cells added was 333 cells). T cell proliferation was measured by $\mathrm{Ki}-67$ expression at day +5 . CAR T cells were gated out by their expression of GFP. Controls included $T$ cells alone (i.e., no proliferation), mock-transduced T cells, and CD19 CAR T cells. These mock-transduced T cells did not express a CAR but were treated in an identical fashion as the transduced CD83 CAR T cells. The CD19 CAR T cells used an identical 41BB costimulation domain as the CD83 CAR T cells, but targeted an irrelevant antigen. One of 2 representative experiments is shown. with CD83 or mock-transduced CAR T cells had no evidence of early xenogeneic GVHD or toxicity compared with PBMCs alone (Figure 4, A and B). However, CD83 CAR T cells significantly improved xenogeneic GVHD survival after transplant, compared with PBMCs alone or mock-transduced CAR T cells (Figure 4A). Additionally, xenogeneic GVHD clinical severity was reduced by CD83-targeted CAR T cells (Figure 4B). Remarkably, mice in both dose cohorts of CD83-targeted CAR T cells demonstrated 3 -month survival of $90 \%$ or better (Figure $4 \mathrm{~A}$ ). In separate experiments, transplanted NSG mice received PBMCs alone or with mock-transduced T cells $\left(1 \times 10^{6}\right)$ or CD83-targeted CAR T cells ( 1 $\left.\times 10^{6}\right)$ and were humanely euthanized at day +21 to evaluate target organ GVHD severity. GVHD path scores were determined by a blinded expert pathologist (41-43). In xenogeneic GVHD models, the recipient lung and liver are critical target organs involved in severe disease (41). CD83 CAR T cells eliminated xenogeneic GVHD target organ tissue damage by human $\mathrm{T}$ cells in the recipient lung (Figure 4, C-E) and liver (Figure 4, G-J), compared with PBMCs alone or mock-transduced $\mathrm{T}$ cells. Moreover, few human $\mathrm{T}$ cells directly infiltrated the murine target organs, and they were not proliferative based on Ki-67 staining (Figure 4, E, F, I, and J). We also tested the preclinical efficacy of human CD83 CAR T cells in GVHD treatment. In these experiments, NSG mice first received human PBMCs with administration of CD83 CAR or mock-transduced $\mathrm{T}$ cells delayed until day +14 when the mice began to show signs of clinical xenogeneic GVHD. The CD83 CAR $T$ cells rescued the mice from xenogeneic GVHD and significantly improved survival (Supplemental Figure 1, A and B; supplemental material available online with this article; https:// doi.org/10.1172/JCI135754DS1).

Human CD83-targeted CAR T cells significantly reduced CD83+ $D C s$ in vivo. Mature CD83+ DCs are implicated in the sensitization of alloreactive donor $\mathrm{T}$ cells. We determined the effect of CD83 CAR T cells on the immune recovery of human CD1 $\mathrm{c}^{+}$
DCs in transplanted mice. NSG mice transplanted with human PBMCs plus CD83 CAR or mock-transduced T cells were euthanized on day +21 . Upon harvesting recipient spleens, we determined that CD83-targeted CAR T cells reduced the expansion of donor cells in vivo, as indicated by much smaller spleens in this treatment group (Supplemental Figure 2). CD83-targeted CAR T cells significantly reduced the amount of human $\mathrm{CD} 1 \mathrm{c}^{+}, \mathrm{CD}^{2} 3^{+}$, and $\mathrm{CD} 1 \mathrm{c}^{+} \mathrm{MHC}$ class $\mathrm{II}^{+} \mathrm{DCs}$ in recipient mice (Figure $5, \mathrm{~A}-\mathrm{C}$ ). However, the proportion of total CD1c $\mathrm{c}^{+}, \mathrm{CD} 14^{-}$DCs among mice treated with CD83 CAR T cells was similar to mice transplanted with PBMCs alone (Figure 5A).

Human CD83-targeted CAR T cells significantly reduced $C D 4^{+}$ and $C D 83^{+} T$ cells while increasing the Treg/activated Tconv ratio in vivo. Using the EGFP tag, we confirmed that infused human CD83 CAR $T$ cells were detectable in murine spleens at day +21 (Figure $6 \mathrm{~A})$. At day +21 , the total amount of human $\mathrm{CD} 4^{+} \mathrm{T}$ cells in the spleens of mice treated with CD83-targeted CAR T cells were significantly reduced (Figure 6, B and C). As we observed significant amounts of $\mathrm{CD} 83^{+}$and $\mathrm{CD} 4^{+}$Tconvs after DC allostimulation in vitro, we confirmed that $\mathrm{CD} 83^{+}$Tconvs were increased at day +21 among mice treated with PBMCs alone or with mock-transduced T cells (Figure 6D). Moreover, the amount of CD83 ${ }^{+}$Tconvs was significantly decreased in recipients of CD83 CAR T cells in vivo (Figure 6D). Overall, the CD83 CAR T cells provided robust elimination of $\mathrm{CD} 83^{+}$target cells by day +21 , compared with mock $\mathrm{T}$ cells (Supplemental Figure 3A). While higher numbers of circulating $\mathrm{EGFP}^{+} \mathrm{CAR} \mathrm{T}$ cells were linked to fewer $\mathrm{CD}^{+} 3^{+} \mathrm{DCs}$ at day +21 , the reduction in $\mathrm{CD}^{+} 3^{+} \mathrm{T}$ cells was uniform across CAR $\mathrm{T}$ cell numbers in vivo (Supplemental Figure 3, B and C). In separate experiments, NSG mice were transplanted with human T cells alone or T cells plus DCs. While the lack of DCs slightly delayed GVHD onset, the median GVHD survival was similar among both groups (Supplemental Figure 4, A and B). This is consistent with work from others showing purified human $\mathrm{T}$ cells are sufficient to 
A

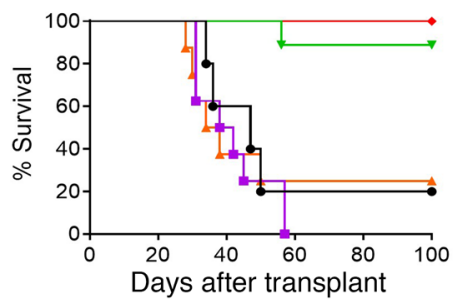

B

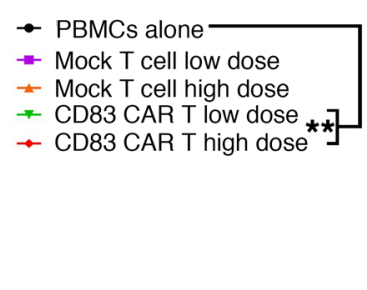

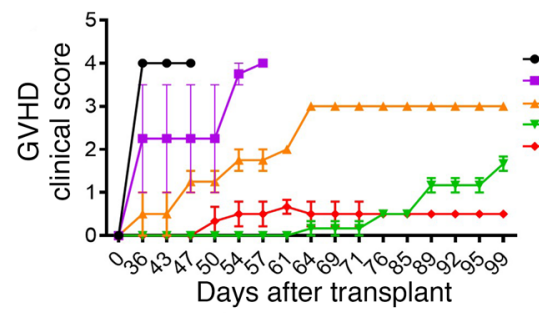
Days after transplant

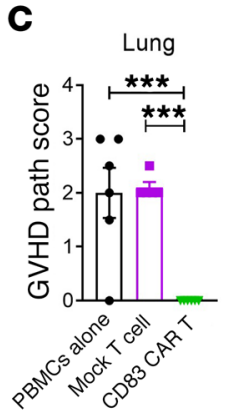

D PBMCs alone
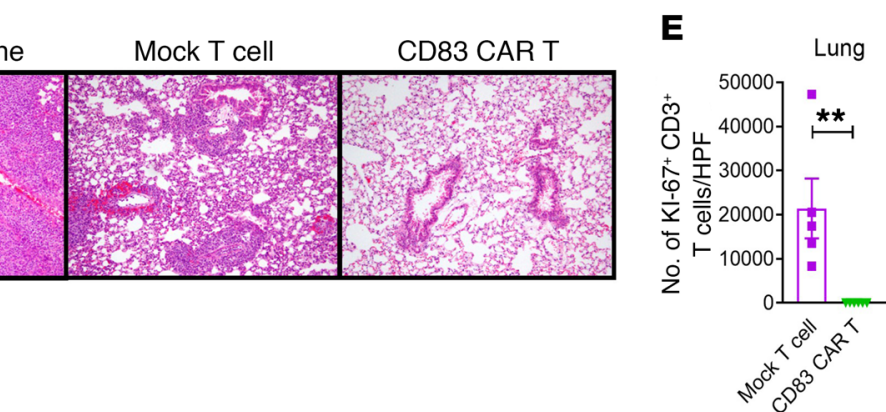

- PBMCs alone

Mock T cell low

* CD83 CAR T low

$\rightarrow$ CD83 CAR T high
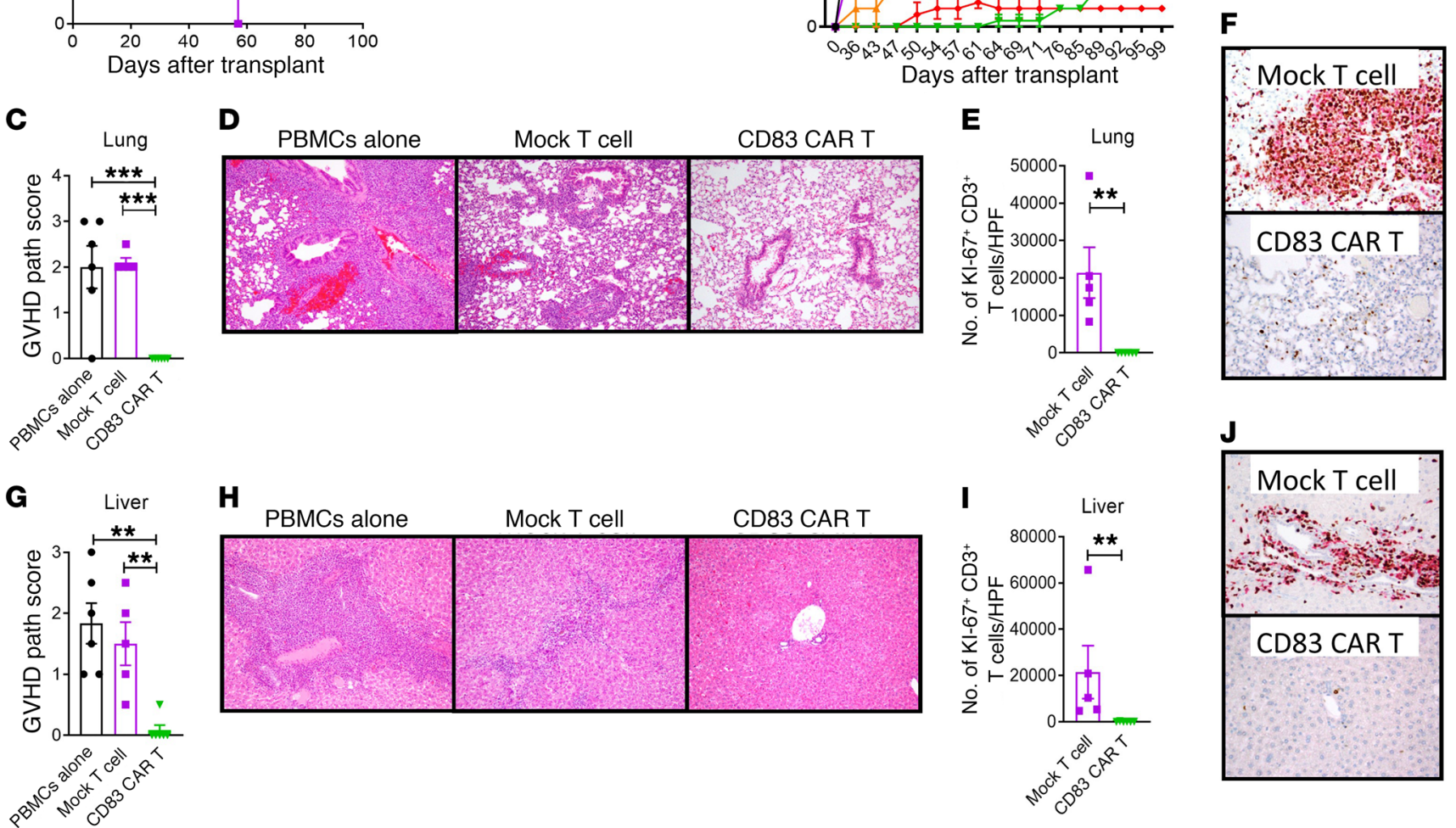

H
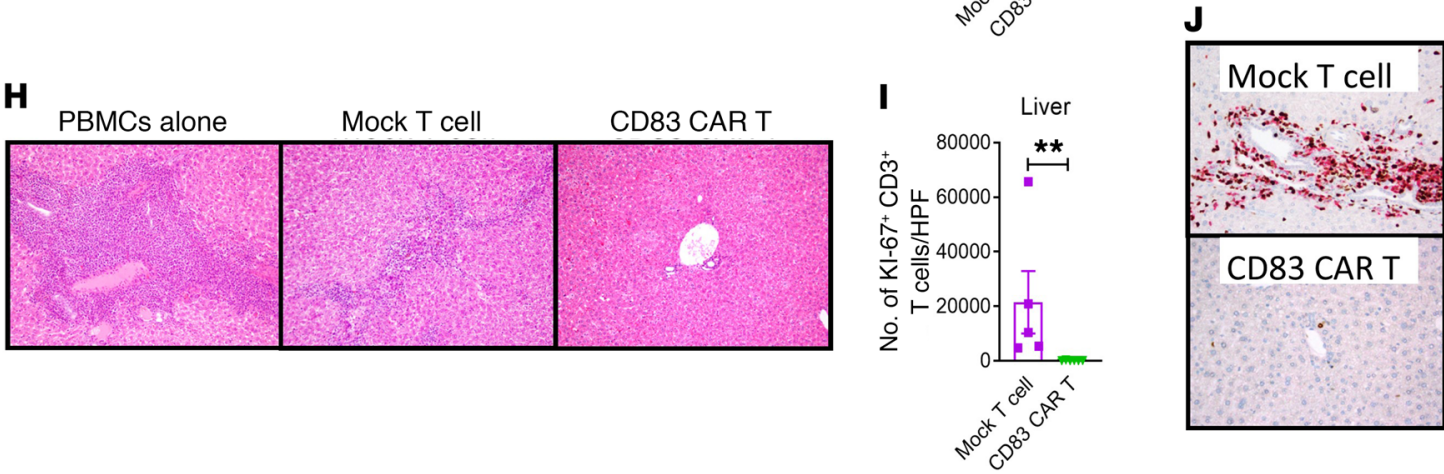

Figure 4. Human CD83 CAR T cells prevent xenogeneic GVHD. (A) NSG mice received $25 \times 10^{6}$ human PBMCs and were inoculated with low $\left(1 \times 10^{6}\right)$ or high dose $\left(10 \times 10^{6}\right)$ CD83 CAR or $\left(1 \times 10^{6}\right.$ to $\left.10 \times 10^{6}\right)$ mock-transduced T cells. The CARs were autologous to the PBMC donor. An additional control group of mice received PBMCs alone. (A) Survival and (B) GVHD clinical scores are shown. Clinical scores incorporate an aggregate assessment of activity, fur and skin condition, weight loss, and posture. Pooled data from 3 independent experiments, up to 9 mice per experimental arm for survival, with a representative experiment depicting long-term GVHD clinical scores. In separate experiments, recipient mice were humanely euthanized at day +21 and tissue GVHD severity was evaluated by an expert blinded pathologist. Xenogeneic GVHD path scores, representative H\&E images, amount of Ki-67+ $\mathrm{CD} 3^{+} \mathrm{T}$ cells/HPF, and representative IHC images (CD3, red; Ki-67, brown) are shown for recipient lung (C-F) and liver (G-J). Original magnification, $\times 100$. Pooled data from 2 independent experiments, up to 6 mice per experimental arm. Log-rank test (A), ANOVA (C and $\mathbf{G})$, Mann-Whitney $(\mathbf{E}$ and $\mathbf{I}) .{ }^{* *} P=0.001-0.01$ and ${ }^{* * *} P=0.0001-0.001$.

induce xenogeneic GVHD (44). In this model, eliminating human DCs alone fails to protect recipients from xenogeneic GVHD.

We surmise that CD83-targeted CAR T cells protect recipients from GVHD primarily by eliminating alloreactive Tconvs implicated in GVHD while enhancing the ratio of Tregs to alloreactive Tconvs (Figure 6, E-G). The frequency of human Tregs in murine spleens was similar among all experimental groups at day +21 (Figure 6E). Like the reduction in total $\mathrm{CD} 4^{+} \mathrm{T}$ cells, the absolute number of Tregs was significantly decreased in mice treated with CD83-targeted CAR T cells (Figure 6F). However, the ratio of Tregs $\left(\mathrm{CD}^{+}, \mathrm{CD}^{2} 7^{-}, \mathrm{CD}^{2} 5^{+}\right.$, Foxp3 $\left.{ }^{+}\right)$to activated Tconvs $\left(\mathrm{CD} 4^{+}, \mathrm{CD} 127^{+}, \mathrm{CD} 25^{+}\right)(41)$ was significantly increased in mice that received CD83 CAR T cells (Figure 6G). Th1 cells and IFN- $\gamma$ contribute to GVHD pathogenesis $(45,46)$. Importantly, mice treated with CD83 CAR T cells exhibited a profound reduction in human $\mathrm{CD} 4^{+} \mathrm{IFN}-\gamma^{+}$Th1 cells (Figure $6, \mathrm{H}$ and I). Additionally, the amount of spleen-resident human Th2 cells $\left(\mathrm{CD} 4^{+}, \mathrm{IL}^{-} 4^{+}\right)$was also significantly decreased in the mice injected with CD83 CAR
T cells (Figure 6, H and J). Conversely, CD83-targeted CAR T cells did not suppress the amount of human Th17 cells (Supplemental Figure 5, A and B) in recipient spleens, compared with PBMCs alone or mock-transduced CAR T cells. We detected EGFP ${ }^{+} \mathrm{CD} 83$ CAR T cells in the spleens of mice surviving to the day +100 endpoint in long-term experiments (Supplemental Figure 6). Over 3 months after transplant, we observed a dose-dependent reduction in circulating $\mathrm{CD}^{2} 3^{+}$target cells among mice treated with a low 1 $\left.\times 10^{6}\right)$ or high $\left(10 \times 10^{6}\right)$ dose of CD83 CAR T cells (Supplemental Figure 6). While CD83 CAR T cells prevent and treat xenogeneic GVHD, they importantly did not impair the proliferation or function of antiviral $\mathrm{CD} 4^{+}$or $\mathrm{CD} 8^{+} \mathrm{T}$ cells responding to clinically relevant infectious peptides, including cytomegalovirus, Epstein-Barr virus, and influenza (Supplemental Figure 7, A and B).

CD83 is a cellular target for human AML. According to longitudinal data from the Center for International Blood and Marrow Transplant Research (CIBMTR), more than 1000 patients receive allo-HCT for high risk AML each year (47). Even when patients can 
A
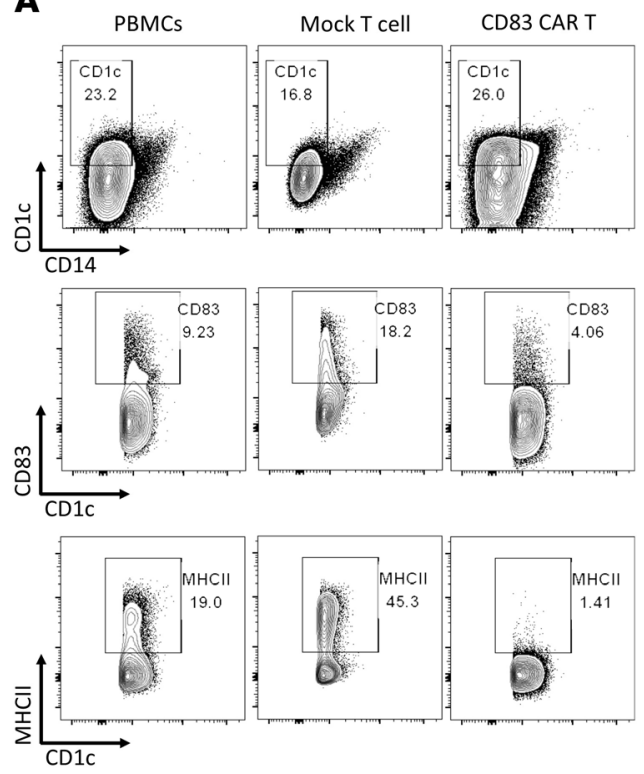

B
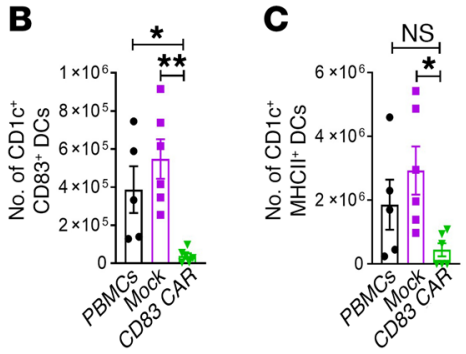

Figure 5. Human CD83-targeted CAR T cells significantly reduce $\mathrm{CD}^{+} 3^{+}$ DCs. NSG mice received $25 \times 10^{6}$ human PBMCs plus $1 \times 10^{6}$ CD 83 CAR or mock-transduced T cells as described. Mice were humanely euthanized on day +21 and the spleens were harvested. (A) Representative contour

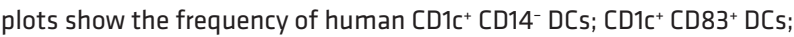
and $\mathrm{CD}^{+} \mathrm{C}^{+} \mathrm{MHC}$ class $\mathrm{II}^{+} \mathrm{DC}$ in the mouse spleens at day +21 . Graph shows the absolute number (mean \pm SEM) of human (B) CD1C ${ }^{+}$CD83 $3^{+}$DCs and (C) $\mathrm{CD}_{1 \mathrm{C}^{+}} \mathrm{MHC}$ class $\mathrm{II}^{+} \mathrm{DC}$ in the mouse spleens at day +21 . Pooled data from 2 independent experiments, up to 6 mice per experimental arm. ANOVA (B and $\mathrm{C}) .{ }^{*} P<0.05 ;{ }^{*} P=0.001-0.01$

tolerate a myeloablative preparative regimen, relapse-free survival is limited to $67.8 \%$, compared with $47.3 \%$ after reduced-intensity conditioning (12). Thus, strategies to prevent AML relapse are needed. Given the potent lytic activity of the CD83 CAR T cell in xenogeneic GVHD, and that AML remains the number one indication for allo-HCT in adults, we investigated whether human myeloid leukemia potentially expressed CD83. We discovered that CD83 is expressed on malignant myeloid K562, Thp-1, U937, and MOLM-13 cell lines (Figure 7, A and B, and Supplemental Figure 8, $\mathrm{A}$ and B). Moreover, CD83 CAR T cells demonstrated significant antitumor activity against $\mathrm{K} 562$ and Thp-1 cells in vitro using the xCELLigence platform (Figure 7, C and D). We also confirmed that CD83 CAR T cells could effectively kill MOLM-13 leukemia in vivo (Figure 7, E and F). Therefore, the human CD83 CAR T cell has the capability to prevent GVHD and provide direct killing of myeloid leukemia. We further compared the expression of CD83 on freshly acquired AML blasts ( $n=15$ patients) to that of CD33 or CD123 (Figure 7, G and H), which are putative myeloid targets for anti-
AML CAR T cells currently in clinical development. The frequency of $\mathrm{CD}^{2} 3^{+}$myeloid blasts was significantly greater than the expression of CD33 or CD123 among patients with AML (Figure 7G).

Human AML antigens are often shared with progenitor stem cells. While CD83 CAR T cells kill myeloid leukemia, we confirmed that they permit the growth and differentiation of hematopoietic stem cells in colony forming units (CFU) assays (Supplemental Figure 9, A-D). However, it should be noted that this CFU assay was performed after a 4-hour coculture of CAR $\mathrm{T}$ cells and stem cells, as described by Gill et al. (48). Therefore, killing of target cells is limited at this time point and may not provide a complete evaluation of potential bone marrow toxicity by targeting CD83. Furthermore, as a first-in-human target, we are evaluating the expression of CD83 in multiple tissues using microarrays (Supplemental Figure 10). Identified CD83 expression among these human tissues will need be evaluated to determine if the cells expressing CD83 are tissue-resident DCs or others (Supplemental Figure 10).

\section{Discussion}

The use of CAR T cells as cellular immunotherapy to prevent GVHD is an innovative strategy, distinct from pharmacologic immune suppression or adoptive transfer of donor Tregs. Targeting cells that express CD83 efficiently depletes transplant recipients of inflammatory mature DCs as well as alloreactive $\mathrm{CD}^{+}$ Tconvs. While human DC depletion did not reduce xenogeneic GVHD, we cannot exclude DC targeting by CD83 CAR T cells as a possible mechanism in GVHD prophylaxis. Further, potential cross-reactivity with murine $\mathrm{CD} 83^{+}$antigen presenting cells could impact the degree of immune suppression in this model. However, the profound depletion of alloreactive $\mathrm{T}$ effectors by CD83 CAR $\mathrm{T}$ cells mediates a significant rise in the Treg/activated Tconv ratio, which is a clinically relevant index in controlling GVHD (49). The kinetics of CD83 expression after CD3/CD28 stimulation and our cytotoxicity assays (Figures 1 and 2) suggest there is potential for $\mathrm{T}$ cell fratricide that could complicate CAR T cell production. In our short 7-day CAR T cell production we did not detect a significant impact on CAR T cell production, but this could be an issue when production is scaled up. Decreased CAR T cell production yields could be mitigated by inclusion of a CD83-blocking antibody during production.

CD83 CAR T cells significantly reduce pathogenic human Th1 and Th2 cells in vivo. This is consistent with the observed high expression of CD83 on human Th1 and Th2 cells, compared with Th17 cells. Experiments using STAT4 and STAT6 knockout donor $\mathrm{T}$ cells have shown that Th1 and Th2 cells independently mediate lethal GVHD in mice (45). Additionally, the combination of Th1 and Th2 cells in vivo cooperatively worsen murine GVHD (45). In part, Th1 and Th2 cells cause tissue-specific damage to the intestine and lungs, respectively (50). During GVHD, T cell-derived IFN- $\gamma$ also mediates apoptosis of intestinal stem cells and related epithelial tissue damage (46). Novel strategies to target donor Th1 responses currently exist and are largely driven by p40 cytokine neutralization or inhibition of relevant downstream receptor signal transduction $(41-43,51,52)$. However, few approaches concurrently target pathogenic Th1 and Th2 cells. Thus, human CD 83 CAR T cells represent what we believe is a novel cell product to 
A

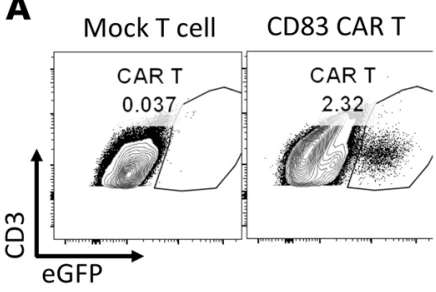

B

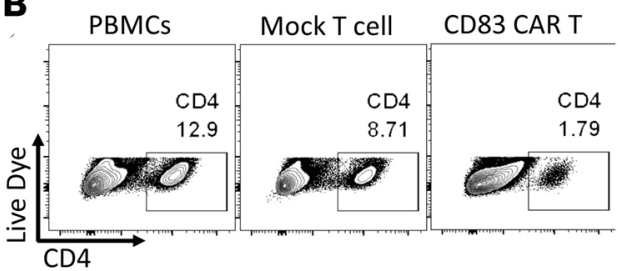

E

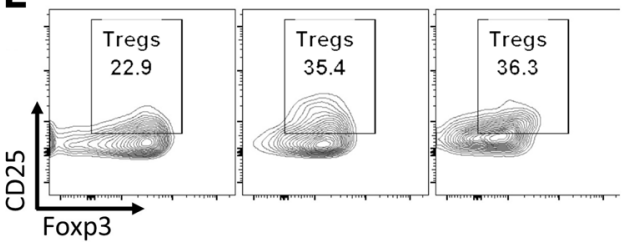

H

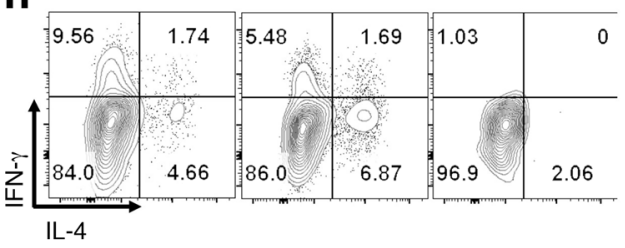

C

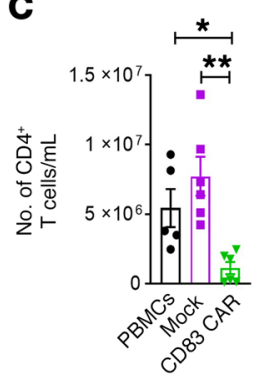

$\mathbf{F}$

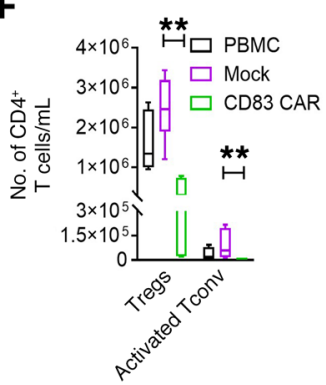

I

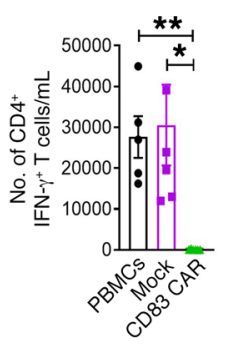

D

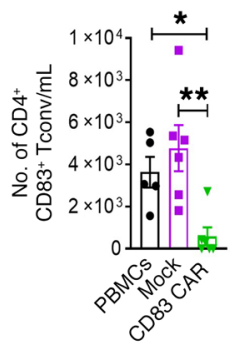

G
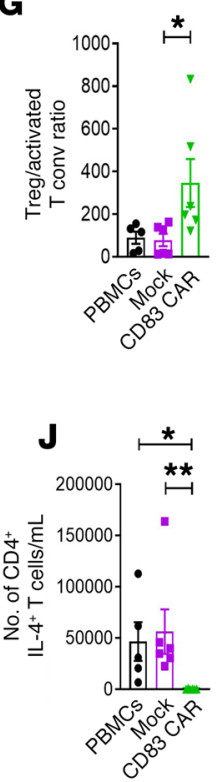

Figure 6. Human CD83-targeted CAR T cells significantly reduce CD4+ and CD83+ $T$ cells while increasing the Treg/activated Tconv ratio in vivo. NSG mice received $25 \times 10^{6}$ human PBMCs plus $1 \times 10^{6}$ CD83 CAR or mock-transduced T cells as described. Mice were humanely euthanized on day +21 and the spleens were harvested. (A) Representative contour plots show the amount of EGFP+ CD83 CAR T cells in the inoculated mice at day +21 , compared with mice that received mock-transduced T cells. (B) Representative contour plots show the frequency of human CD4+ $T$ cells in the recipient spleens. Graphs show the absolute numbers (mean \pm SEM) of (C) $\mathrm{CD}^{+}$and (D) $C D 4^{+}$CD83 ${ }^{+} \mathrm{T}$ cells in the mouse spleens at day +21 . (E) Contour plots depict the percentage of CD4 ${ }^{+}$, $\mathrm{CD}_{127}, \mathrm{CD}^{+} 5^{+}, \mathrm{Foxp3}^{+}$Tregs in the mouse spleens at day +21 . Graphs show the amount (mean \pm SEM) of (F) Tregs and the (G) Treg/activated Tconv ratio at day +21 in the recipient mice. (H) Contour plots depict the frequency of CD4+ IFN- $\gamma^{+}$Th1 cells and CD4+ IL- $4^{+}$Th2 cells in the mouse spleens at day +21. Graphs demonstrate the absolute numbers (mean \pm SEM) of (I) Th1 and (J) Th2 cells in the recipient spleens. Pooled data from 2 independent experiments, up to 6 mice per experimental arm. ANOVA (C, D, F, G, I, and J). ${ }^{*} P<0.05,{ }^{* *} P=0.001-0.01$.

simultaneously suppress donor Th1 and Th2 responses after alloHCT. We found that human Th17 cells express very little CD83 and were largely unaffected by CD83 CAR T cells, though the treated mice were clearly protected from GVHD. While donor Th17 cells have the potential to contribute toward GVHD (53), the lack of available Th1 cells could theoretically mitigate the pathogenicity of the surviving Th17 cells (52).

Our data support that human CD83 CAR T cells provide durable protection from activated Tconvs and GVHD mortality. Though CD83 is not significantly expressed on human Tregs, mice treated with human CD83 CAR T cells exhibited reduced amounts of Tregs. This may be due to limited availability of CD4 ${ }^{+}$ $\mathrm{T}$ cells for peripheral Treg induction or diminished IL-2 concentrations by the overall reduction in circulating donor T cells. In rodents, CD83 participates in Treg stability in vivo and mice bearing CD83-deficient Tregs are susceptible to autoimmune syndromes (54). However, in our xenotransplantation experiments the ratio of human Tregs to activated Tconvs was significantly increased in mice treated with CD83 CAR T cells compared with controls. The increased ratio of Tregs to Tconvs is a clinically relevant immune indicator, and even correlates with response to Treg-directed GVHD therapy such as low-dose IL-2 $(49,55)$. Moreover, the human CD83 CAR T cells were well tolerated and eliminated immune-mediated organ damage in vivo. Thus, the role of CD83 may differ among murine and human Tregs.

CD83 is a unique immune regulatory molecule. In mice, soluble CD83 mediates immune-suppressive effects by enhancing Treg responses through indoleamine 2,3-dioxygenase and TGF- $\beta$ mechanisms (56). The extracellular domain of human CD83 was also shown to impair alloreactive $\mathrm{T}$ cell proliferation in vitro (57). Conversely, direct neutralization of CD83 with monoclonal antibody $3 \mathrm{C} 12 \mathrm{C}$ significantly reduced xenogeneic GVHD mediated by human $\mathrm{T}$ cells in vivo (33). The anti-CD83 antibody also preserved Tregs and antiviral responses by donor human $\mathrm{CD}^{+} \mathrm{T}$ cells (34). 
A

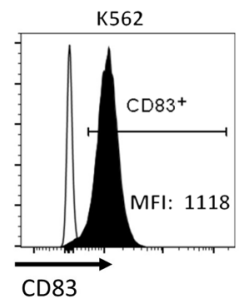

C

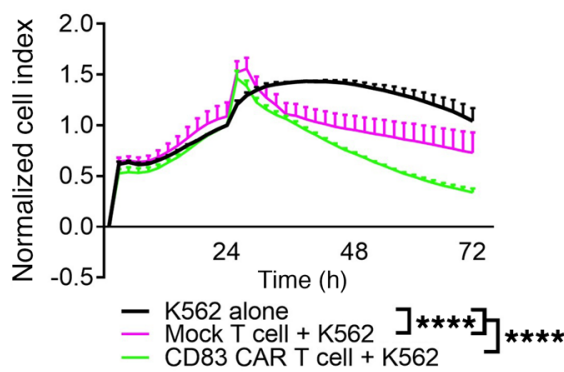

D

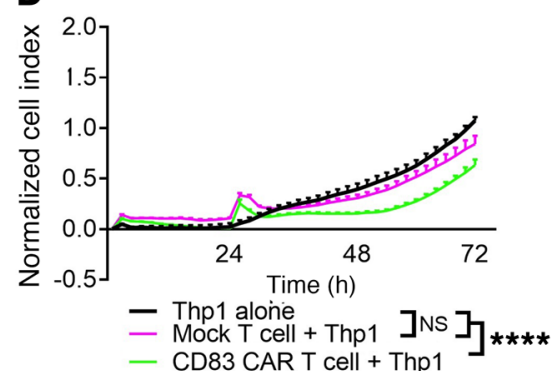

$\mathbf{E}$

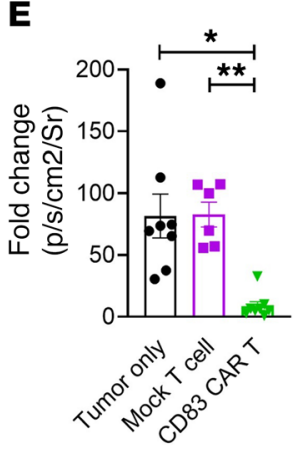

$\mathbf{F}$

Pre-CAR

Tumor only

B

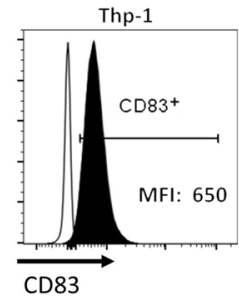

Figure 7. CD83 is a cellular target for human AML. Histograms show CD83 expression among proliferating (A) K562 and (B) Thp-1 cells with MFI noted in the lower right-hand corner (FMO, unfilled). Human CD83 CAR or mock-transduced T cells were cocultured with fresh $\mathrm{K} 562$ or Thp-1 cells at an E/T ratio of 10:1. (C and $\mathbf{D})$ Target cell killing was monitored using the $x$ CELLigence RTCA system. A representative experiment for each is shown (triplicate mean \pm SEM). NSG-SGM3 mice were injected with MOLM-13 EGFP/luciferase ${ }^{+} 1 \times 10^{6}$ cells. Bioluminescence (BLI) was performed (IVIS Systems) on day +9 and then the mice were treated with $2.5 \times 10^{6}$ CD83 CAR T or mock-transduced T cells. Mice were then imaged weekly. (E) Graph shows fold change (mean \pm SEM) for average radiance $(p /$ $\mathrm{sec} / \mathrm{cm}^{2} / \mathrm{Sr}$ ) at 1 week after injection of CD83 CAR or mock-transduced T cells. (F) Representative image shows weekly BLI intensities among each mouse per group over 4 weeks ( $n$ $=2$ independent experiments, with 7-8 mice per experimental arm). (G) Graph shows the expression (mean \pm SEM) of CD83 compared with CD33 or CD123 among freshly acquired human $\mathrm{CD} 4^{+} \mathrm{AML}$ blasts. (H) Representative histogram shows CD83 expression among human CD34+ AML blasts (FMO, unfilled) ( $n=$ 15 patient samples). ANOVA (C, D, E, and $\mathbf{G}) .{ }^{*} P$ $<0.05,{ }^{* *} P=0.001-0.01,{ }^{* * *} P=0.0001-0.001$, and ${ }^{* * *} P<0.0001$.

Week 2

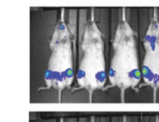

Week 1

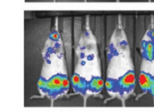

Week 3

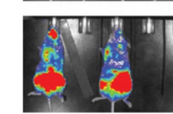

Week 4

H

\section{G}

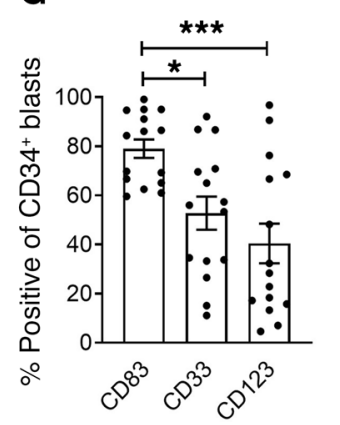

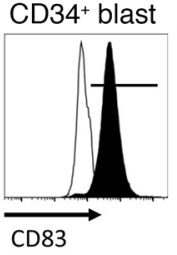

This suggests that while soluble CD83 may have immune suppressive properties, targeting the cell surface expression of CD83 can prevent GVHD while retaining key effector and Treg function. Distinct from a monoclonal antibody, CD83 CAR T cells elicit robust target cell killing alone; without the need for NK cell-mediated antibody-dependent cellular cytotoxicity (34). This is an advantage when rapid, efficient elimination of alloreactive $\mathrm{T}$ cells is needed to prevent or treat GVHD. Indeed, human CD83-targeted CAR T cells provided lasting GVHD prophylaxis and were detectable in mice up to day +100 even after a single infusion. Moreover, upcoming phase IB clinical trial in relapsed/refractory AML.

AML antigens are often expressed on progenitor stem cells, and we show robust CD83 expression on blasts from donors with AML. Therefore, we evaluated for stem cell killing in human CFU assays, which demonstrated negligible on-target, off-tumor toxicity. Thus, CD83 CAR T cells permit normal hematopoiesis after coculture of CD83-targeted CAR T cells and stem cells for 4 hours. However, this early time point may incompletely evaluate potential bone marrow toxicity due to limited time for cytotoxic killing by the CD83 CAR T cells. As a first-in-human target we are per- 
forming an extensive safety evaluation of CD83 for an Investigational New Drug application. We started by characterizing expression of CD83 in tissue microarrays (Supplemental Figure 10). Considering the limitation of the CFU assays, we will also evaluate for bone marrow toxicity in immune-deficient mice engrafted with human hematopoietic stem cells followed by infusion of CD83-targeted CAR T cells. Thus, the current level of evidence in our present work cannot make definitive claims regarding the potential safety of the CD83 CAR T cells. That important aspect of these CD83 CAR T cells must be evaluated in a well-designed phase I trial. However, potential risks for off-tumor, on-target toxicity mediated by CD83 CAR T cells can likely be mitigated by gene-engineering strategies such as RNA-based CAR gene transfer, drug regulation of CAR expression, suicide gene inclusion, stem cell genome editing, or logic gating.

Allo-HCT is often necessary to treat high risk AML, though relapse remains an important cause of posttransplant failure and death. Distinct from HLA-mediated classic GVL, CD83 CAR T cells selectively kill CD83-expressing malignant cells. Moreover, it was recently discovered that CD83 is also expressed on Hodgkin lymphoma (HL) (58). Therefore, the CD83 CAR T cells may have efficacy in treating AML or HL independent of allo-HCT. This is translationally powerful, given the clinical success of CD19 CAR T cells in ALL and diffuse large B cell lymphoma (26-29).

In conclusion, CD83 CAR T cells represent the first human, programmed cytolytic effector cell designed to prevent or treat GVHD. We demonstrate the translational potential of CD83 CAR T cells in managing GVHD, though we expect it to have merit in preventing rejection after solid organ or vascularized composite allograft transplantation, too. Furthermore, CD83 CAR T cells retain their killing activity even when exposed to calcineurin inhibitors. We hypothesize that CD83 CAR T cells will overcome barriers of HLA disparity in hematopoietic cell and solid organ donor selection, and greatly extend the application of curative transplantation procedures to patients in need. Importantly, CD83 CAR T cells provide a platform to eliminate alloreactive $\mathrm{T}$ cells without the need for broadly suppressive, nonselective calcineurin inhibitors or glucocorticoids. Moreover, CD83 CAR T cells preserve donor antiviral immunity against CMV, EBV, and influenza. The ability of CD83 CAR T cells to kill myeloid leukemia cells further extends its clinical impact. Unlike existing GVHD prophylaxis options that aim to preserve GVL at best, the CD83 CAR T cell has the potential to concurrently prevent GVHD as well as actively target life-threatening AML relapse. Thus, the CD83 CAR T cell carries high likelihood to reduce transplantrelated mortality and improve outcomes after allo-HCT.

\section{Methods}

CD83 CAR T cell construct and production. The CD83 CAR was synthesized and cloned into SFG retroviral construct by GENEWIZ (35, 36). The CD 83 SFG-cloned construct was transfected into H29 cells using calcium phosphate, and retroviral supernatants were used to transduce RD114. Retroviral supernatant of RD114 cells was filtered through $0.45 \mu \mathrm{m}$ strainer (MilliporeSigma). CD83 CAR T cells were generated by transduction of human $\mathrm{T}$ cells as described $(35,36)$. Briefly, gammaretrovirus was produced using H29- and RD114producing cell lines, and certain aliquots were titered against NIH/3T3 cells. Leukocytes obtained from apheresis from a healthy human donor (All Cells) were isolated by density gradient centrifugation. $\mathrm{T}$ cells were isolated using magnetic beads (Stem Cells Inc.) and stimulated with human CD3/CD28 activation beads (Thermo Fisher Scientific) in RPMI with recombinant human IL-2. Activated T cells were transduced with gamma retrovirus on RetroNectin-coated (TaKaRa Bio Inc.) plates. For all viral transduction, $1 \mathrm{~mL}$ virus was incubated with $1 \mathrm{~mL}$ T cells. CD83 CAR T cells were debeaded after 7-8 days. Gene transfer or transduction efficiency was estimated by $\mathrm{GFP}^{+}$cells using flow cytometry, given the CAR construct contains an intracellular EGFP reporter (Figure 1, A and B).

Monoclonal antibodies and flow cytometry. Fluorochrome-conjugated mouse anti-human monoclonal antibodies included anti-CD3, -CD4, -CD8, -CD25, -CD45, -CD83, -CD1c, -CD127, -MHCII, -Foxp3, -Ki-67, -IFN- $\gamma$, -IL-17A, and -IL-4 (BD Biosciences, eBioscience, Cell Signaling Technology) (Supplemental Table 1). LIVE/DEAD Fixable Yellow or Aqua Dead Cell Stain (Life Technologies) was used to determine viability. Live events were acquired on a BD FACSCanto II or LSRII flow cytometer (FlowJo software, version 7.6.4, Tree Star).

Cytokine immunoassays. CD83 CAR and mock-transduced T cells $\left(1 \times 10^{5}\right)$ were cocultured with CD83 ${ }^{+}$moDCs $\left(1 \times 10^{4}\right)$ for 24 hours. Supernatants were harvested and analyzed using a human luminex assay kit (R\&D Systems) on a Luminex 100 system and Simple Plex Assay Kit (Biotechne) on an Ella instrument (Biotechne). Manufacturers' instructions were followed (36).

Human CD83 CAR T cell cytotoxicity and in vitro proliferation. Normalized CD83 CAR T cells $\left(1 \times 10^{5}\right.$ cells $)$ were cultured with CD83 ${ }^{+}$ moDCs, activated T cells, K562, or Thp-1 cells at an E/T ratio of 5:1 or 10:1 in duplicates in E-Plate 96 (ACEA Biosciences Inc.). Cytotoxicity assay was run on an xCELLigence RTCA (real-time cell analysis) instrument (ACEA Biosciences) according to the manufacturer's instructions. Similarly, human CD83 CAR T cells were cocultured with moDCs at an $\mathrm{E} / \mathrm{T}$ ratio of 1:1 in non-tissue-culture-treated 6-well plates in triplicate. Cells were grown in human T cell complete medium supplemented with $60 \mathrm{IU} / \mathrm{mL}$ IL-2. Cell viability and total cell numbers in each well were measured on days $+1,+7$, and +14 on a cell counter (Bio-Rad) with trypan blue staining.

In vitro alloMLRs. Human moDCs were cytokine-generated, differentiated, and matured as described (41). A quantity of $10^{5} \mathrm{~T}$ cells purified from leukocyte concentrates (OneBlood or Memorial Blood Center) were cultured with allogeneic moDCs (T cell/DC ratio 30:1) in $100 \mu \mathrm{L}$ complete RPMI supplemented with $10 \%$ heat-inactivated pooled human serum (41-43). Third-party CD83 CAR, CD19 CAR, or mock-transduced $\mathrm{T}$ cells were added to the alloMLR at a range of CAR-to-DC ratios. $\mathrm{T}$ cell proliferation was measured after 5 days by Ki-67 expression.

CD83 expression on human $T$ cells (time course) and bone marrow blasts. Purified human T cells were stimulated with either allogeneic moDCs ( $\mathrm{T}$ cell/DC ratio 30:1) or CD3/CD28 beads ( $\mathrm{T}$ cell/bead ratio 30:1). T cells were harvested from triplicate wells in a 96-well plate at $4,8,24$, and 48 hours of culture. T cells were stained for CD3, CD4, CD127, CD25, and CD83, then fixed. CD83 expression was evaluated in activated Tconvs $\left(\mathrm{CD}^{+}, \mathrm{CD}^{+}, \mathrm{CD}^{2} 7^{+}, \mathrm{CD} 25^{+}\right)(41)$, Tregs $\left(\mathrm{CD}^{+}, \mathrm{CD}^{+}, \mathrm{CD}^{2} 7^{-}, \mathrm{CD} 25^{+}\right)(41)$, and $\mathrm{CD} 8 \mathrm{~T}$ cells $\left(\mathrm{CD}^{+}, \mathrm{CD}^{-}\right)$. Where indicated, CD83 CAR or mock T cells were cultured with DCallostimulated PBMCs, and CD83 expression was evaluated among the $\mathrm{CD}^{-}$and $\mathrm{CD}^{+}$target cells over 48 hours. Deidentified, bank bone 
marrow samples from healthy donors $(n=10)$ and patients $(n=10)$ with active AML were acquired from University of Minnesota Malignancies Tissue Bank. The frozen marrow samples were viably thawed and stained for live dead aqua, anti-human CD45, CD117, CD34, and CD83. CD83 expression was determined by isotype control on CD34 ${ }^{+}$ myeloid blasts.

Colony-forming units. CFU assays were performed as described by Gill et al. (48). CD $34^{+}$cells isolated from normal human bone marrow were purchased from All Cells. A quantity of $10^{3}$ cells were cocultured with either CD83-targeted CAR T cells, mock T cells, or media alone. Cells were incubated for 4 hours at an E/T ratio of 10:1. Following incubation, cells were plated in MethoCult medium (StemCell) in 6-well SmartDish plates (StemCell) according to the manufacturer's instructions and cultured for 14 days. At the end of the culture period, colonies were imaged, analyzed, and counted using the STEMvision software.

Xenogeneic GVHD model. Recipient NSG mice received $25 \times$ $10^{6}$ fresh human PBMCs (OneBlood) once on day 0 of the transplant. As indicated, mice either received PBMCs alone, PBMCs plus CD83 CAR T cells (low dose, $1 \times 10^{6}$; high dose, $10 \times 10^{6}$ ), or PBMCs plus mock-transduced T cells $\left(10 \times 10^{6}\right)$. Each independent experiment was performed with a different human PBMC donor, where the CAR T cells and mock-transduced $\mathrm{T}$ cells were derived from the PBMC donor. Mice were monitored for GVHD clinical scores and premoribund status. Where indicated, short-term experiments were completed on day +21 via humane euthanasia to evaluate blinded GVHD target organ pathology, tissue-resident lymphocytes, and the content of human DCs and T cell subsets within the murine spleens (41-43). Tissue samples were prepared, stained (Ventana Medical Systems), and imaged (Vista) to identify human $\mathrm{Ki}^{2} 7^{+} \mathrm{T}$ cells as previously described (41). These mice were transplanted with PBMCs $\left(25 \times 10^{6}\right)$ with or without CD83 CAR T cells $\left(1 \times 10^{6}\right)$ or mock-transduced T cells $\left(1 \times 10^{6}\right)$. Murine in vivo data were pooled from at least 2 independent experiments with 6-9 mice per experimental group.

In vivo bioluminescence experiments. NSG-SGM3 mice were injected with $1 \times 10^{6}$ MOLM-13 EGFP/Luciferase ${ }^{+}$cells. Bioluminescence (BLI) was performed (IVIS Systems) on day +9 and then the mice were injected with $2.5 \times 10^{6} \mathrm{CD} 83$ CAR or mock-transduced $\mathrm{T}$ cells. Mice were then imaged weekly for up to 4 weeks.

Statistics. Data are reported as mean values \pm SEM. ANOVA was used for group comparisons, including a Sidak's or Dunn's post test for correction of multiple comparisons. Mann-Whitney was used for all others. For comparison of survival curves, a log-rank test was used.
The statistical analysis was conducted using Prism software version 5.04 (GraphPad). Statistical significance was defined by a 2-tailed $P$ less than 0.05 .

Study approval. NSG mice (male or female, age 6-24 weeks old) were purchased from Jackson Laboratory and housed within one of the American Association for Laboratory Animal Care's accredited animal resource centers at Moffitt Cancer Center or the University of Minnesota. All mice were treated in adherence with the NIH's Guide for the Care and Use of Laboratory Animals (National Academies Press, 2011) and the protocols used were approved by local institutional animal care and use committees. All vertebrate animal work was performed under an AICUC-approved protocol.

\section{Author contributions}

MLD and BCB contributed equally in the design and performance of experiments, analysis and interpretation of data, and writing of the manuscript. MLD designed and produced the CD83 CAR T cell. BS performed experiments, analyzed and interpreted data, and edited the manuscript. KW, JR, JB, NT, GL, and TG performed experiments. EMS performed histologic and pathologic analyses of xenograft data and edited the manuscript. MF and JSM assisted in the design and analysis of human AML samples. JP participated in translational development of the CD83 CAR T cell and edited the manuscript. $\mathrm{BRB}$ and $\mathrm{CA}$ designed experiments and edited the manuscript.

\section{Acknowledgments}

Our study received assistance from the Flow Cytometry Core Facility at the H. Lee Moffitt Cancer Center \& Research Institute (P30CA076292) and the Flow Cytometry Resource at the University of Minnesota, both of which are NCI-designated Comprehensive Cancer Centers. The authors would like to acknowledge the animal care staff in the Department of Comparative Medicine at the University of South Florida for providing technical assistance. This work was supported by funds from the Moffitt Cancer Center (to MLD); NIH grants K08 HL116547 (to BCB), R01 HL133823 (to BCB), R01 HL11879, R01 HL56067, and R37 AI34495 (to BRB); and Leukemia \& Lymphoma Society Translational Research Grant 6462-15 (to BRB).

Address correspondence to: Marco L. Davila, 12902 Magnolia Drive, Tampa, Florida 33612, USA. Email: marco.davila@moffitt. org. Or to: Brian C. Betts, Nils Hasselmo Hall, Room 2-108, 312 Church Street, SE Minneapolis, Minnesota 55455, USA. Email: bett0121@umn.edu.
1. Cutler C, et al. Tacrolimus/sirolimus vs tacrolimus/methotrexate as GVHD prophylaxis after matched, related donor allogeneic HCT. Blood. 2014;124(8):1372-1377.

2. Pidala J, et al. A randomized phase II study to evaluate tacrolimus in combination with sirolimus or methotrexate after allogeneic hematopoietic cell transplantation. Haematologica. 2012;97(12):1882-1889.

3. Zeiser R, et al. Inhibition of CD4+CD25+ regulatory $\mathrm{T}$-cell function by calcineurindependent interleukin-2 production. Blood. 2006;108(1):390-399.

4. Powles RL, Barrett AJ, Clink H, Kay HE, Sloane
J, McElwain TJ. Cyclosporin A for the treatment of graft-versus-host disease in man. Lancet. 1978;2(8104-5):1327-1331.

5. Storb R, et al. Marrow transplantation for severe aplastic anemia: methotrexate alone compared with a combination of methotrexate and cyclosporine for prevention of acute graft-versus-host disease. Blood. 1986;68(1):119-1251.

6. Storb R, et al. Methotrexate and cyclosporine compared with cyclosporine alone for prophylaxis of acute graft versus host disease after marrow transplantation for leukemia. $\mathrm{N} \mathrm{Engl} \mathrm{JMed.}$ 1986;314(12):729-735.

7. Pidala J, et al. Nonpermissive HLA-DPB1 mis- match increases mortality after myeloablative unrelated allogeneic hematopoietic cell transplantation. Blood. 2014;124(16):2596-2606.

8. Anasetti C, et al. Peripheral-blood stem cells versus bone marrow from unrelated donors. $N$ Engl J Med. 2012;367(16):1487-1496.

9. Sorror ML, et al. Comparing morbidity and mortality of HLA-matched unrelated donor hematopoietic cell transplantation after nonmyeloablative and myeloablative conditioning: influence of pretransplantation comorbidities. Blood. 2004;104(4):961-968.

10. Thakar MS, et al. The Hematopoietic Cell Transplant Comorbidity Index predicts survival 
after allogeneic transplant for nonmalignant diseases. Blood. 2019;133(7):754-762.

11. Solh MM, Solomon SR, Morris LE, Zhang X, Holland HK, Bashey A. The dilemma of conditioning intensity: when does myeloablative conditioning improve outcomes for allogeneic hematopoietic cell transplantation. Biol Blood Marrow Transplant. 2019;25(3):606-612.

12. Scott BL, et al. Myeloablative versus reducedintensity hematopoietic cell transplantation for acute myeloid leukemia and myelodysplastic syndromes. J Clin Oncol. 2017;35(11):1154-1161.

13. Bolaños-Meade J, et al. Three prophylaxis regimens (tacrolimus, mycophenolate mofetil, and cyclophosphamide; tacrolimus, methotrexate, and bortezomib; or tacrolimus, methotrexate, and maraviroc) versus tacrolimus and methotrexate for prevention of graft-versus-host disease with haemopoietic cell transplantation with reduced-intensity conditioning: a randomised phase 2 trial with a non-randomised contemporaneous control group (BMT CTN 1203). Lancet Haematol. 2019;6(3):e132-e143.

14. Veerapathran A, Pidala J, Beato F, Yu XZ, Anasetti C. Ex vivo expansion of human Tregs specific for alloantigens presented directly or indirectly. Blood. 2011;118(20):5671-5680.

15. Veerapathran A, et al. Human regulatory T cells against minor histocompatibility antigens: ex vivo expansion for prevention of graft-versushost disease. Blood. 2013;122(13):2251-2261.

16. Walton $\mathrm{K}$, et al. Metabolic reprogramming augments potency of human pSTAT3-inhibited iTregs to suppress alloreactivity. JCI Insight. 2020;5(9):136437.

17. Brunstein CG, et al. Infusion of ex vivo expanded $\mathrm{T}$ regulatory cells in adults transplanted with umbilical cord blood: safety profile and detection kinetics. Blood. 2011;117(3):1061-1070.

18. Brunstein CG, et al. Umbilical cord bloodderived T regulatory cells to prevent GVHD: kinetics, toxicity profile, and clinical effect. Blood. 2016;127(8):1044-1051.

19. Kellner JN, et al. Third party, umbilical cord blood derived regulatory $\mathrm{T}$-cells for prevention of graft versus host disease in allogeneic hematopoietic stem cell transplantation: feasibility, safety and immune reconstitution. Oncotarget. 2018;9(86):35611-35622.

20. Ruggeri L, et al. Effectiveness of donor natural killer cell alloreactivity in mismatched hematopoietic transplants. Science. 2002;295(5562):2097-2100.

21. Olson JA, Leveson-Gower DB, Gill S, Baker J, Beilhack A, Negrin RS. NK cells mediate reduction of GVHD by inhibiting activated, alloreactive T cells while retaining GVT effects. Blood. 2010;115(21):4293-4301.

22. Asai O, et al. Suppression of graft-versus-host disease and amplification of graft-versus-tumor effects by activated natural killer cells after allogeneic bone marrow transplantation. J Clin Invest. 1998;101(9):1835-1842.

23. $\mathrm{Du}$ J, et al. Invariant natural killer $\mathrm{T}$ cells ameliorate murine chronic GVHD by expanding donor regulatory T cells. Blood. 2017;129(23):3121-3125.

24. Highfill SL, et al. Bone marrow myeloid-derived suppressor cells (MDSCs) inhibit graft-versushost disease (GVHD) via an arginase-1-dependent mechanism that is up-regulated by interleukin-13. Blood. 2010;116(25):5738-5747.

25 . Bruce DW, et al. Type 2 innate lymphoid cells treat and prevent acute gastrointestinal graftversus-host disease. J Clin Invest. 2017;127(5):1813-1825.

26. Neelapu SS, et al. Axicabtagene ciloleucel CAR $\mathrm{T}$-cell therapy in refractory large B-cell lymphoma. NEngl JMed. 2017;377(26):2531-2544.

27. Schuster SJ, et al. Tisagenlecleucel in adult relapsed or refractory diffuse large B-cell lymphoma. N Engl J Med. 2019;380(1):45-56.

28. Maude SL, et al. Tisagenlecleucel in children and young adults with B-cell lymphoblastic leukemia. N Engl J Med. 2018;378(5):439-448.

29. Davila ML, et al. Efficacy and toxicity management of 19-28z CAR T cell therapy in B cell acute lymphoblastic leukemia. Sci Transl Med. 2014;6(224):224ra25.

30. Ghosh A, et al. Donor CD19 CAR T cells exert potent graft-versus-lymphoma activity with diminished graft-versus-host activity. Nat Med. 2017;23(2):242-249.

31. Ju X, et al. The analysis of CD83 expression on human immune cells identifies a unique CD83+-activated T cell population. JImmunol. 2016;197(12):4613-4625.

32. Koyama M, et al. Donor colonic CD103+ dendritic cells determine the severity of acute graft-versus-host disease. J Exp Med. 2015;212(8):1303-1321.

33. Wilson J, et al. Antibody to the dendritic cell surface activation antigen CD83 prevents acute graft-versus-host disease. J Exp Med. 2009;206(2):387-398.

34. Seldon TA, et al. Immunosuppressive human anti-CD83 monoclonal antibody depletion of activated dendritic cells in transplantation. Leukemia. 2016;30(3):692-700.

35. Li G, Park K, Davila ML. Gammaretroviral production and $\mathrm{T}$ cell transduction to genetically retarget primary T cells against cancer. Methods Mol Biol. 2017;1514:111-118.

36. Li G, et al. 4-1BB enhancement of CAR T function requires NF- $\mathrm{KB}$ and TRAFs. JCI Insight. 2018;3(18):121322.

37. Szabolcs P, et al. Dendritic cells and macrophage can mature independently from a human bone marrow-derived, post-colony-forming unit intermediate. Blood. 1996;87(11):4520-4530.

38. Krzyzak L, et al. CD83 modulates B cell activation and germinal center responses. J Immunol. 2016;196(9):3581-3594.

39. Lechmann M, Shuman N, Wakeham A, Mak TW. The CD83 reporter mouse elucidates the activity of the CD83 promoter in B, T, and dendritic cell populations in vivo. Proc Natl Acad Sci USA. 2008;105(33):11887-11892.

40. Betts BC, et al. Janus kinase-2 inhibition induces durable tolerance to alloantigen by human dendritic cell-stimulated $\mathrm{T}$ cells yet preserves immunity to recall antigen. Blood. 2011;118(19):5330-5339.

41. Betts BC, et al. Targeting Aurora kinase A and JAK2 prevents GVHD while maintaining Treg and antitumor CTL function. Sci Transl Med. 2017;9(372):eaai8269.

42. Betts BC, et al. Targeting JAK2 reduces GVHD and xenograft rejection through regulation of T cell differentiation. Proc Natl Acad Sci USA. 2018;115(7):1582-1587.

43. Betts BC, et al. Inhibition of human dendritic cell ER stress response reduces $\mathrm{T}$ cell alloreactivity yet spares donor anti-tumor immunity. Front Immunol. 2018;9:2887.

44. Li W, et al. Proteomics analysis reveals a Th17-prone cell population in presymptomatic graft-versus-host disease. JCI Insight. 2016;1(6):86660.

45. Nikolic B, Lee S, Bronson RT, Grusby MJ, Sykes M. Th1 and Th2 mediate acute graft-versus-host disease, each with distinct end-organ targets. JClin Invest. 2000;105(9):1289-1298.

46. Takashima S, et al. T cell-derived interferon- $\gamma$ programs stem cell death in immunemediated intestinal damage. Sci Immunol. 2019;4(42):eaay8556.

47. Gupta V, Tallman MS, Weisdorf DJ. Allogeneic hematopoietic cell transplantation for adults with acute myeloid leukemia: myths, controversies, and unknowns. Blood. 2011;117(8):2307-2318.

48. Gill S, et al. Preclinical targeting of human acute myeloid leukemia and myeloablation using chimeric antigen receptor-modified T cells. Blood. 2014;123(15):2343-2354.

49. Koreth J, et al. Interleukin-2 and regulatory $\mathrm{T}$ cells in graft-versus-host disease. $N$ Engl J Med. 2011;365(22):2055-2066

50. Yi T, et al. Reciprocal differentiation and tissue-specific pathogenesis of Th1, Th2, and Th17 cells in graft-versus-host disease. Blood. 2009;114(14):3101-3112.

51. Pidala J, et al. In vivo IL-12/IL-23p40 neutralization blocks Th1/Th17 response after allogeneic hematopoietic cell transplantation. Haematologica. 2018;103(3):531-539.

52. Yu Y, et al. Prevention of GVHD while sparing GVL effect by targeting Th1 and Th17 transcription factor T-bet and ROR $\gamma \mathrm{t}$ in mice. Blood. 2011;118(18):5011-5020.

53. Iclozan $\mathrm{C}$, et al. T helper17 cells are sufficient but not necessary to induce acute graft-versushost disease. Biol Blood Marrow Transplant. 2010;16(2):170-178.

54. Doebbeler M, et al. CD83 expression is essential for Treg cell differentiation and stability. JCI Insight. 2018;3(11):99712.

55. Koreth J, et al. Efficacy, durability, and response predictors of low-dose interleukin-2 therapy for chronic graft-versus-host disease. Blood. 2016;128(1):130-137.

56. Bock F, et al. Topical application of soluble CD83 induces IDO-mediated immune modulation, increases Foxp3+ T cells, and prolongs allogeneic corneal graft survival. J Immunol. 2013;191(4):1965-1975.

57. Lechmann M, et al. The extracellular domain of CD83 inhibits dendritic cell-mediated T cell stimulation and binds to a ligand on dendritic cells. J Exp Med. 2001;194(12):1813-1821.

58. Li Z, et al. CD83 is a new potential biomarker and therapeutic target for Hodgkin lymphoma. Haematologica. 2018;103(4):655-665. 